\title{
EL CONOCIMIENTO RELACIONAL DEL SINGULAR EN ERNST CASSIRER
}

\author{
GUSTAVO ESPARZA
}

Universidad Panamericana

\begin{abstract}
RESUMEN: Este documento profundiza en la propuesta de solución sobre el conocimiento del singular desarrollado por Ernst Cassirer. El problema plantea que la relación entre lo particular y lo general se concibe ya en los primeros estudios del autor hasta cristalizar en su propuesta de intercambiar los conceptos sustantivos por conceptos funcionales. Este intercambio metodológico permitirá al neokantiano fundamentar, eventualmente, (1) la Philosophie der Symbolischen Formen como cosmovisión que concibe a la cultura como criterio universal de sentido de todas las acciones del ser humano y (2) al símbolo como unidad funcional armónica entre el singular y el universal.
\end{abstract}

PALABRAS CLAVE: singular; universal; funcionalismo; simbolización; cultura; Cassirer.

\section{The relational knowledge of singular on Ernst Cassirer}

ABSTRACT: This paper deepens the proposed solution on the knowledge of the singular developed by Ernst Cassirer. The problem arises that the relationship between the particular and the general is already conceived in the first studies of the author until crystallizing in his proposal to exchange substantive concepts for functional concepts. This methodological exchange will allow the neo-Kantian to base, eventually, (1) the Philosophie der Symbolischen Formen as a worldview that conceives culture as a universal criterion of meaning of all human actions and (2) the symbol as a harmonic functional unit between the singular and the universal.

KEY WORDS: singular; universal; functionalism; symbolization; culture; Cassirer.

\section{INTRODUCCIÓN}

La relación entre el singular y el universal (S/U) constituye una preocupación permanente dentro de la obra del filósofo neokantiano Ernst Cassirer ${ }^{1}$. Este aspecto puede constatarse desde sus trabajos tempranos. En su disertación doctoral sobre la filosofía cartesiana, el estudio de lo infinito (das Unendlichen) se presenta como la síntesis entre la pluralidad de la experiencia y la conceptuación propia del pensa-

1 En relación a las abreviaturas de las obras de Cassirer, aunque existe una edición de obras completas [Ernst Cassirer Gesammelte Werke. Hamurger Ausgabe, 2009], no se ha determinado con unanimidad una forma de citación canónica. Una forma de citación de sus obras corresponde a la que a continuación se propone y se toma en cuenta en este trabajo para las obras mayores: SuF (CASSIRER, 1910/1953, Substanzbegriff und Funktionsbegriff). PSF (CASSIRER, Philosophie der symbolischen Formen) PSF, I (CASSIRER, 1923/1998. Die Sprache), PSF, II (CASSIRER, 1924/1998. Das mythische Denken), PSF, III (CASSIRER, 1929/1998. Phänomenologie der Erkenntnis) y PSF, IV (CASSIRER, 1995/1996. Metaphysiche der Symbolischen Formen), AEM, (CASSIRER, 1945. An essay on man). 
miento $^{2}$. En este sentido, la cuestión fundamental que expone el autor es la valoración de posibilidades para articular la multiplicidad de los sucesos en un concepto que opere como representación universal de los recursos sintetizados. Al respecto el filósofo de Breslavia considerará que no hay tales posibilidades en la propuesta cartesiana ya que para Descartes la finitud humana es incapaz de integrar la infinitud del mundo (Cassirer 1902, p. 77) ${ }^{3}$.

Con el paso de la filosofía cartesiana al pensamiento leibniziano, el neokantiano concibe un sistema con en el cual lograr la síntesis de lo diverso. Concretamente para Cassirer (1902) el trabajo de Leibniz consistirá en la posibilidad de conjuntar la infinitud de datos singulares y los conceptos universales a través de una ley general constitutiva ${ }^{4}$. El conjunto de estas ideas se sintetiza en el trabajo histórico $L a$ filosofía de la Ilustración en donde expondrá la teoría básica del conocimiento del singular:

[En la filosofía de Leibniz encontramos] el principio de continuidad... Continuidad significa unidad en la multiplicidad, ser en el devenir, permanencia en el cambio; una conexión que no puede expresarse más que en el cambio y el continuo transformarse de determinaciones y para la cual, por lo tanto, la diversidad se exige tan necesariamente y de modo tan radical y esencial como la unidad. La relación entre lo universal y lo particular se presenta a una nueva luz... no existe entro lo universal y lo particular una pura relación de subsunción. Porque no se trata tan solo de subordinar este a aquel, sino que hay que conocer cómo está implicado y fundado en él (Cassirer 1975, pp. 46-47. Énfasis en el original.).

De este modo, para el filósofo de Breslavia aparecerá como un factor necesario la reflexión sobre los recursos y procesos a través de los cuales es posible la conciliación de las unidades singulares en una relación de universalidad sin con ello perder su identidad propia. Esta es, a decir del propio autor, la complejidad

2 De acuerdo con el propio CASSIRER (1993, p. 449, nota 2) su tesis doctoral apareció publicada en 1899, y utilizada como introducción a su disertación sobre Leibiniz. Presentamos a continuación un fragmento que refleja los planteamientos iniciales sobre el problema del singular: «Die notwendige Beziehung von Erfahrung und Denken, die uns allgemein in den Grundlagen der neueren Wissenschaft entgegentritt, hat sich in keinem ihrer Begriffe charakteristischer ausgeprägt, als im Begriff des Unendlichen. Denn das Unendliche vertritt die Eigenart der reinen Denkfunktion selbst, deren Wert und Notwendigkeit es gegenüber den Einwänden der populären und sinnlichen Auffassung erweist» (CASSIRER 1902, p. 77).

3 El neokantiano vuelve a insistir en estas ideas en el primer volumen de El problema del conocimiento: «El mismo Descartes, en su réplica a las objeciones de Gassendi, expresa con palabras que en punto a fuerza no se quedan por debajo de las de Galileo, el criterio de que jamás podremos llegar a obtener un conocimiento perfecto y exhaustivo, no ya de lo infinito, pero ni siquiera de un fragmento concreto de la realidad, por circunscrito y diminuto que sea» (CASSIRER 1993, p. 487).

${ }_{4}$ En su trabajo sobre Leibiniz, el neokantiano considerará como paradigma de reflexión la forma de la ciencia como modelo clave de reflexión: "Auch das Denken der Wissenschaft muss zunächst noch notwendig von begrifflichen Bestimmungen ausgehen, die es als festen und gesicherten Besitz betrachtet. [...] Nach ihr sollen nicht nur die Dinge aus den Ideen abgeleitet werden; vor allem dürfen auch die einzelnen ideellen Voraussetzungen nicht als abgeschlossene, sich selbst genügende Daten gelten. Die Mannigfaltigkeit der Erkenntnisse und Wahrheiten muss aus einer ursprünglichen Einheit des Gesetzes begriffen und erschaffen warden» (CASSIRER 1902, pp. 218-219). 
central que daría origen al desarrollo de su primera gran obra sistemática $S u F$, en el prólogo a ella, el autor anota: «The investigations contained in this volume were first prompted by studies in the philosophy of mathematics. In the course of an attempt to comprehend the fundamental conceptions of mathematics from the point of view of logic, it became necessary to analyse more closely the function of the concept itself and to trace it back to its presuppositions» (SuF, p. iii. Énfasis añadido.).

El intercambio entre los conceptos sustantivos por los conceptos funcionales, a decir de Cassirer, implicará que todo acto de conocimiento prescinde del interés por captar directamente las sustancias para, en su lugar, emprender un conocimiento a través de la intermediación simbólica. Este planteamiento ha sido interpretado como un idealismo cuyo reducto niega la posibilidad del conocimiento de lo real y, por ende, la aceptación de que no es posible la aprehensión de lo singular ${ }^{5}$. Contrario a esta idea, la filosofía de las formas simbólicas (en tanto que proyecto de investigación) mantiene como meta ultima del conocimiento el conocimiento del ser:

Parece que nosotros sólo podemos captar [la] realidad en la peculiaridad de [las] formas... La función del pensamiento no debe reducirse a «expresar» el ser... el pensamiento se siente capaz de hacer frente a la realidad; alberga la convicción y cree poseer la seguridad de que puede agotar su contenido. Aquí no debe ni puede seguir habiendo ninguna barrera infranqueable, pues el pensamiento y el objeto al cual el primero se dirige son una misma cosa (PSF, III, p. 12).

De este modo, el conocimiento del singular ya no se alcanza por medio del conocimiento de lo sustancial sino por medio de la mediación funcional de los conceptos. Ahora, en lugar de establecer que entre ambos media una relación dispar y sustancialmente incompatible, el conocimiento reorienta su punto de acceso al conocimiento de la realidad reubicando el problema del modo en que se vinculan lo percibido inmediato con un concepto. Cassirer considera que «el objeto de la experiencia, sólo es posible bajo el supuesto del entendimiento y sus funciones a priorísticas unificadoras» $(P S F$, III, p. 16). En este camino, lo universal se articula con lo singular como parte de una «coordinación funcional» (Ryckman 1991, pp. 62-75) de sentido o, como el autor eventualmente las llamará, «formaciones simbólicas» (PSF, I-III).

\footnotetext{
5 Los principales opositores a esta supuesta falta de una metafísica realista se encuentran en el volumen colectivo coordinado por Paul A. SchiLpp (1949) y más actualmente Leon RosensteIn (1973, p. 314-319) ha cuestionado las posibilidades de alcanzar una Metaphysics of Symbolic Forms en el sentido en que el neokantiano la ha prometido en PSF, IV. Contrario a estos planteamientos, Donald P. VERENE (2008, p. 93-103) argumenta dos ideas: primera, los señalamientos de una oposición a la metafísica se sostiene de suponer que el neokantismo tiene como único referente valido la ciencia, idea errónea y atribuible a una falta de conocimiento profundo de los planteamientos sistemáticos por parte de la Escuela de Marburgo y sobre todo de las formas simbólicas como una filosofía de la «función simbólica» y no específicamente de los símbolos por separado; segundo, gran parte de las críticas compiladas por Schilpp (1949) se dirigen únicamente a una lectura de la PSF y no así al conjunto de la obra del filósofo de Breslavia, lo que explicaría por qué existe una radical diferencia en los puntos de vista.
} 
A partir de este breve recorrido, el presente trabajo profundiza en las operaciones lógicas, así como en las operaciones psicológicas a través de las cuales las formaciones simbólicas permiten el conocimiento relacional del singular en el conjunto de la obra de Cassirer. La principal aportación se centra en mostrar la paulatina argumentación de cómo la función matemática es concebida como un recurso que permite (a) conjuntar las unidades singulares, en cuanto realidades aprehendidas, (b) en el marco de una ley general que les dota de significado dentro de una estructura universal.

\section{LA FORMA Y FUNCIÓN CIENTÍfICA DEL CONOCIMIENTO EN CASSIRER}

Si quisiéramos caracterizar la filosofía de Ernst Cassirer de algún modo, la primera tentativa apuntaría a presentarla como «neokantiana», pero sería más preciso considerarla como una Filosofía de las formas simbolicas. El interés por delimitar la tradición del filósofo de Breslavia es relevante porque la propia comprensión del enfoque metodológico con que el neokantiano trata los temas, constituye una primera aproximación a la solución del problema que aquí se estudia ${ }^{6}$. En términos generales su obra elucida si los símbolos (lenguaje, mito y ciencia, entre otros) son un agregado de la cultura o si, por medio de ellos esta se diversifica y específica con un carácter peculiar e independiente. Si los símbolos operaran como agregados de las expresiones y actividades culturales, entonces, cada forma simbólica constituiría un fragmento de la totalidad y tendrían que integrarse para configurar una imagen completa de mundo en la que se desarrolla el ser humano, de este modo se requiere una relación cuantitativa de los elementos singulares $(a+b+c \ldots+n)$ para conocer la universalidad $(\mathrm{N})^{7}$. Si, por el contrario, los símbolos se consideran como una expresión peculiar e independiente, habría que considerar a cada forma como un «índice de refracción» (PSF, III, p. 14) en donde los elementos singulares se conocen por su relación funcional $(\varphi[\mathrm{a}, \mathrm{b}, \mathrm{c}])$. La respuesta, aunque constituiría una fundamentación lógica, ofrecería el conjunto de recursos metodológicos con los

6 Para una revisión de la tradición y los problemas para definir la tradición de Cassirer: (FERRARI 2010).

7 María García Amilburu (1998) ha profundizado este aspecto haciendo notar que toda la filosofía de Cassirer se puede entender como una filosofía de la cultura que tiene como base un análisis crítico del conjunto de las formas que la integran, para ella el neokantiano parte de la realidad como un hecho fenoménico cuyo signo es interpretado. A través de esta hermenéutica del signo, la cultura se convierte en un universo simbólico dinámico toda vez que los contextos de sentido se apoyan de las relaciones de significado en las que el conjunto de interacciones humanas ocurre. Esta tesis de la autora puede confirmarse con el trabajo de Sebastian LuFT (2004) quien propone que la filosofía Cassirer es una «Fenomenología hermenéutica» (Hermeneutic phenomenology) ya que se interesa por los modos en que la conciencia interpreta el mundo. Por su parte, Wolfgang Овтн (2011) aclara aún más esta idea cuando escribe: "Culture" for Cassirer thus designates the vital, active relation of the human being to the world as well as the world itself. The world is manifest to the human being, as is the problem of the "knowledge of reality" ( (ORTH 2011, p. 117). El neokantiano desarrolla estas ideas en dos trabajos principalmente: (CASSIRER, 1979, pp. 64-91) y AEM (pp. 87-96). 
cuales solventar distintos problemas epistemológicos, antropológicos y metafísicos $(A E M ; P S F, \mathrm{IV})^{8}$.

Como deudor de la tradición neokantiana en la Introducción a PSF, I (pp. 12$60)$, Cassirer se pregunta si una crítica de la razón le podría ofrecer un camino favorable para la investigación y solución a este problema concluyendo que tal vía resultaría insuficiente. El neokantiano subraya la necesidad de expandir los términos de la crítica de la razón a la cultura, para con ello valorar las formas (simbólicas) de expresión espiritual en cuanto a su «ley interna» y a la relación que guarda con aquel contexto simbólico (PSF, I, pp. 17-26). Al respecto Sirkku Ikonen (2011) considera que la pretendida insuficiencia de la razón y su expansión a la cultura no son proyectos excluyentes. Los términos en los que Cassirer propone la tarea tienen su fundamento en la filosofía kantiana, de tal forma que, la crítica de la cultura cassireriana se fundamenta en los términos expuestos por Kant:

Critique, as Cassirer understands it, is not bound up with conceptual conditions of knowledge. Instead of pure categories, the philosophy of symbolic forms is looking for the «basic formative principles» of the specific symbolic forms. These principles do not have to be conceptual, but they vary from one symbolic form to another. Conceptual or theoretical knowledge is only one of the symbolic forms, and the critical method is in no way constrained only to the conceptual symbolic function» (Ikonen 2011, p. 200).

En este sentido, entonces, el vínculo entre lo S/U se constituye como un elemento necesario y natural en el proceso del conocimiento, ya que considera que, entre la advertencia expresiva del mundo, su representación y su cumbre en la simbolización, persiste una relación permanente entre los fenómenos singulares y la ley universal con la que formalmente se interpreta como objeto conocido.

Todo el esfuerzo filosófico de Cassirer se centra en la comprensión de las condiciones del conocimiento y desde su primera gran obra sistemática Substanzbegriff und Funktionsbegriff, esta fijará como característica necesaria del conocimiento su forma relacional. En la obra, el autor plantea que un análisis de la evolución histórica de la ciencia permitiría establecer un camino progresivo en el que se revela la naturaleza y forma del concepto. Este último es el producto intelectual por medio del cual se integran la pluralidad de características esenciales que definen al objeto estudiado, sin embargo, el problema central no se encuentra en la consideración del "concepto» como el producto universal en donde se abstraen las características particulares de los objetivos, sino en considerar a la «abstracción» como el proceso

8 El trabajo de Roberto GonzálEz (2013) elabora una reflexión estudiando las consecuencias de esta afirmación que aquí se sostiene. El autor plantea que en la Filosofía de las formas simbólicas «se opera una confección metodológica... a través de la cual se lleva a efecto la integración o reunión de las diferentes ideas del hombre. Es así que se abre el marco para integración de todas las formas de ser del hombre, reconociendo sus especificidades sin violentar ninguna idea antropológica» (GonZÁlez 2013, p. 19). El principal resultado de esta «confección metodológica» es la capacidad dinámica del ser humano para conocer el mundo por medio de las formas simbólicas humanas, sin por ello, necesariamente asumirse como el creador del mismo mundo que conoce. Esto implica que el símbolo ofrece una doble dirección epistemológica: permite conocer la realidad en su constitución y al ser humano como constructor de símbolos culturales. 
psicológico mediante el cual se alcanza la «conceptuación» ${ }^{9}$. El autor lo plantea del siguiente modo:

Thus, analysis of the theory of abstraction leads back to a deeper problem. The "comparison» of contents... is primarily only a vague and ambiguous expression, which hides the difficulty of the problem. In truth, very different categorical functions are here united under what is merely a collective name. And the real task of logical theory with regard to any definite concept consists in setting forth these functions in their essential characteristics and in developing their formal aspects. The theory of abstraction obscures this task since it confuses the categorical forms, upon which rests all definiteness of the content of perception, with parts of this very content itself $(S u F$, p. 16).

El obscurecimiento, según Cassirer, corresponde al criterio mediante el cual se determina que una característica singular se constituye como característica esencial y, por tanto, universal. Según el autor, el pensamiento abstracto no se ofrece como criterio de construcción de conceptos, toda vez que depende del sujeto el considerar una acción como característica universal de los objetos singulares. Sin embargo, la alternativa propuesta por el autor en primera instancia parece recurrir a un principio similar para la construcción de los conceptos, ya que para el neokantiano es necesaria la postulación de una Ley o sistema general que permita la identificación de las unidades particulares dentro de una relación serial; es decir, la singularidad no se constituye como un objeto que se hace presente por medio de los sentidos, sino como el resultado de una operación de vinculación entre una ley universal y el conjunto de casos singulares que satisfacen el criterio ${ }^{10}$.

Frente a esta aparente contradicción en la que se considera que la abstracción no opera como el recurso psicológico que ofrece la universalización de los casos singulares, el neokantiano aducirá que el concepto científico no se constituye por la negación de las características que, a criterio del investigador, deban evitarse, sino que recae en la metodología experimental propuesta por la ciencia lo que permite descartar las intuiciones del investigador. El carácter universal alcanzado no se refiere a una abstracción de criterios que engloben los rasgos similares de los objetos, sino a la relación de una ley general que permita incorporar el conjunto de casos particulares que satisfacen el principio estipulado.

En este contexto, Cassirer distinguirá entre la «forma de la serie» y los «miembros de la serie» en donde el primer término se refiere a la Ley general de sentido en

$9 \quad$ No es intención del presente trabajo exponer la oposición que Cassirer (SuF, pp. 3-26) plantea a la teoría aristotélica del conocimiento. El neokantiano parte de la consideración de los conceptos sustantivos no pueden ofrecer una vía pertinente de la naturaleza del conocimiento aduciendo, entre otros argumentos, una detallada historia de las ciencias como reflejo del abandono de los conceptos sustantivos y el intercambio por una metodología funcionalista (SuF, pp. 112-233). El filósofo de Breslavia está convencido de que el desarrollo y evolución de las Ciencias Naturales se debe, principalmente, a la consideración de los conceptos funcionales. Para una revisión más detallada: PSF, III (pp. 331-555).

10 Cassirer lo plantea del siguiente modo: «The concept, however, is not deduced thereby, but presupposed; for when we ascribe to a manifold an order and connection of elements, we have already presupposed the concept, if not in its complete form, yet in its fundamental function» $(S u F$, p. 17). 
la que se pueden incluir el conjunto de casos singulares o miembros que satisfacen el criterio general presentado ( $S u F$, pp. 18-26). El autor, al respecto escribe: «The meaning of the law that connects the individual members is not to be exhausted by the enumeration of any number of instances of the law; for such enumeration lacks the generating principle that enables us to connect the individual members into a functional whole» $(S u F$, p. 26). Para el neokantiano, entonces, el conocimiento de las unidades singulares y su relación con la universalidad del concepto se encuentra en el acto sintético de la definición y no de la intuición sensible.

Cassirer (SuF, pp. 309ss.) no deja de reconocer que, con este planteamiento relacional, se sobreviene un problema ontológico toda vez que se pregunta si las relaciones entre los miembros individuales de una serie y su coordinación funcional a una forma general de la serie, constituyen un estatuto del ser o son meras construcciones del pensamiento ${ }^{11}$. La respuesta general aducida en $S u F$ sostiene que el conocimiento se enmarca de un modo claro y determinante dentro de las configuraciones científicas del mundo. Esta misma idea será retomada, posteriormente, en el tercer volumen de su PSF. En esta obra, el neokantiano fija la necesidad de considerar la «construcción» del conocimiento en una función cualitativa en lugar de una función cuantitativa:

[S]e puede siempre [interpretar cualquier fenómeno] como una «abstracción»a partir de los datos senso-intuitivos inmediatamente dados, a fin de reducirlo en última instancia a una mera suma de tales datos de acuerdo con la tesis dominante sobre la esencia de la abstracción. Un principio de explicación de la naturaleza, empero, independientemente de su estructura particular pertenece ya a otro ámbito de validez por razón de su mera dimensión lógica general. Tal principio no se expresa en un concepto, sino en un juicio, en un enunciado general, y todo enunciado de este tipo entraña un modo específico de aserción. Su relación con el mundo de los fenómenos intuitivos es enteramente indirecta a través del medio del «significado». El sentido del principio tiene que verificarse en última instancia empíricamente y, por tanto, intuitivamente, pero esa verificación no es nunca posible directamente, sino que sólo puede llevarse a cabo deduciendo de la hipótesis de su validez otros enunciados mediante una deducción hipotética ( $P S F$, III, p. 334$)^{12}$.

En este pasaje, Cassirer ubica la necesidad de construir un conocimiento universal atribuible a los datos singulares aprehendidos. La construcción de un «principio» que opere como la ley general a través de la cual sea posible identificar los

11 Sobre los postulados metafísicos en CASSIRER: (PSF, IV); (GonZález 2015, pp. 9-56; pp. 103-146).

12 Dimitry Gawronsky resalta esta diferencia entre funciones citando el siguiente encuentro entre Herman Cohen y Ernst Cassirer: «...you put the center of gravity upon the concept of relation [...] the concept of relation is a category; yeti $t$ is a category only insofar as it is function, and function unavoidably demands the infinitesimal element in which alone the root of the ideal reality can be found»(GAWRONSKY 1949, p. 21). La principal diferencia está en que, mientras que Cassirer acepta que la síntesis de los conceptos que conforman a un fenómeno es la base para la construcción de un problema, para Cohen los conceptos son integraciones infinitas de experiencias singulares. Para el filósofo de Breslavia, la magnitud intensiva no representa totalidades por lo que un concepto no es una integración de experiencias particulares sino su relación (EsPaRZA 2017, pp. 59-73). 
datos individuales percibidos por el ser humano. Para cada dato percibido el investigador debe ajustarlo a un marco ya sea teórico o histórico que le permita interpretar una sensación singular como un "signo ${ }^{13}$ específico del cual derivar un sentido. El conocimiento de lo particular, entonces, se convierte en un proceso de identificación por medio de una "coordinación funcional» (Ryckman 1991, pp. 5761), en ella, los elementos singulares se vinculan a una forma general de sentido, lo que permite identificar cada dato como perteneciente a una ley a partir de la cual se les puede reconocer bajo esa directriz espiritual.

Las posibilidades de identificación y significación del singular son posibles mientras se consideren como unidades pertenecientes a una universalidad. En ese sentido, Cassirer investigará las condiciones que determinan que una pluralidad de fenómenos es susceptible de valorarse como unidad plural relacionada. Una primera respuesta se centra en el análisis de cómo la Matemática y la Física habían logrado establecer una teoría del «concepto» (PSF, III, pp. 331-367) y de "grupo» $(P S F$, III, pp. 417-471; Cassirer 1979, pp. 271-291) en tanto que principios de universalización de los elementos singulares.

Al estudiar el problema del «concepto»Cassirer se preguntará «¿Cómo podría existir [alguna] comunidad cuando se trata no de comparar un objeto con otros, sino de constituir, de llegar a la idea de $u n$ objeto individual?» (PSF, III, p. 338). Frente al problema, el autor plantea que la característica distintiva del «concepto» parte de una universalidad a través de la cual se avanza para determinar el conjunto de unidades singulares que satisfacen las condiciones lógicas con las cuales se posibilita la vinculación de cualquier dato concreto con un concepto general que lo explica. Así, parece que el concepto de «grupo» es aquel conjunto de miembros singulares de la serie que pueden atribuirse como pertenecientes a una forma general, sin embargo, el propio autor considera que la reunión grupal constituye un problema específico que no se solventa aduciendo una vinculación funcional entre un miembro particular y su forma general constituye por principio al grupo.

Durante su periodo de trabajo en la Universidad de Yale, Cassirer sostendrá que para lograr la agrupación era necesaria una operación intelectual en la cual establecer una coordinación funcional entre los aspectos individuales percibidos o intuidos con un sistema general que les dote de significado. La necesidad de reunirlos como parte de una relación general que determine que el conjunto de todos los miembros de una serie pertenece a una forma general, no se agota sólo con justificar una cierta relación entre un elemento particular y una ley o sistema ${ }^{14}$.

13 Para una explicación del «signo» y el "símbolo» en Cassirer se recomienda la introducción del propio neokantiano (PSF, I, pp. 12-60), así como los trabajos de Carl HaMBURG (1949) y Roberto GonzÁlez (2013, pp. 41-56).

${ }_{14}$ El autor lo plantea en los siguientes términos: «There must be a reason for this universal applicability of the concept to so many fields of inquiry that, at first sight, seem to have little in common, or even to deal with quite disparate subjects» (CASSIRER, 1979, p. 274). Incluso ofrecerá algún criterio inicial propuesto por la matemática que sería el siguiente: «a group is a set of operations having the property that when two operations are carried out in succession the result is one that would be reached by a single operation of the set. Such a set of operations is called a closed set and the property of closure is called the "group property». The combination —or, as it is usually called, the product - of any two elements $a$ and $b$ of this set, whether $a$ and $b$ are here distinct elements or the same element, is a unique element 
Aunque no pudo terminar de resolver este problema, es claro que Cassirer insistió en que la construcción del conocimiento no se podía reducir al sujeto o al objeto, sino que debe satisfacer un conjunto de criterios lógicos y metafísicos que permiten determinar la relación entre lo individual y lo general.

El filósofo de Breslavia, en el marco de estas consideraciones, elabora un recurso de investigación con el que fuese posible el estudio del «ser» en su forma y carácter universal, pero que diera cuenta de su cotidianeidad singular. El centro de su filosofía está constituido en la indagación de un problema que se enmarca en la relación a la solución del problema ¿Cómo es posible designar algún tipo de característica general cuando encontramos entidades particulares? El autor, al respecto, propone:

El punto de partida de la especulación filosófica está caracterizado por el concepto de ser. En el momento en que este concepto se constituye como tal y en que frente a la multiplicidad y diversidad de los entes despierta la conciencia de la unidad del ser, surge por primera vez la dirección específicamente filosófica de la contemplación del mundo (PSF, I, p. 12).

Parecería, entonces, que hemos llegado nuevamente al punto de partida, al problema de la pluralidad de los singulares y las condiciones que facultan su conocimiento. Sin embargo, el problema se presenta ahora bajo una nueva perspectiva toda vez que parcialmente se responde aduciendo que toda investigación científicofuncional permite explicar la relación entre el ente singular y la forma general del ser. Esta es una primera solución que todavía asume la crítica de la razón pura kantiana como un camino suficiente para la investigación del conocimiento. Eventualmente, Cassirer abre la crítica de la razón a la cultura por considerar que sólo de esta forma sería posible dar cuenta de las distintas expresiones espirituales que hacían posible el conocimiento (PSF, I, pp. 17-26) ${ }^{15}$.

\section{LA ESTRUCTURA Y LA FORMA DEL SISTEMA DE RELACIONES FUNCIONALES EN CASSIRER}

Una de las novedades que propone la Phänomenologie der Erkenntnis es la caracterización y prioridades de las ciencias físico-matemático y las novedades que las investigaciones sobre la mecánica cuántica y la teoría de la relatividad habían

c which is an element of the set $a b=c »$ (CASSIRER, 1979, pp. 274-275). Sin embargo, acepta que esta definición de grupo satisfice sólo algunos casos, por lo que no es posible determinar la universalidad de esta operación. El problema de una búsqueda universal, se verá más adelante, le lleva a preguntarse si lo universal debe buscarse en la forma del conocimiento considerando formaciones distintas a las de la ciencia.

15 El trabajo de IKonen (2011) permite sostener que, a pesar de que la crítica kantiana apertura de la investigación de las formas racionales a las formas de expresión, el proyecto original considerado por el de Konigsberg se mantiene. Entre el proyecto de Kant y el de Cassirer, la metodología crítica sigue operando como factor de relación, pero al partir del «método trascendental», en los términos expuestos por Paul NatorP (1987), el de Breslavia considerará que ahora, las investigaciones de las posibilidades del conocimiento, toda vez que amplia «el propio concepto fundamental de teoría» (PSF, III, p. 7) para demostrar que es posible establecer una simetría lógica entre consecuencias naturales e intelectuales o símbolos en formas no-científicas. 
aportado a la constitución del conocimiento (Kauffman 1949). Sin embargo, el tercer volumen de su obra magna, además de estos avances anteriores reconocerá que la forma y estructura del concepto se ve ampliada al reconocer como campo de validez epistemológica aquellos motivos naturales que ya existen en el mundo natural y que operan bajo un principio intrínseco peculiar:

no sólo son auténticos factores y motivos formales los que imperan en la configuración de la imagen científica del mundo, sino también los que ya existen en la configuración de la «imagen natural mundo», la imagen de la percepción y la intuición (PSF, III, p. 7).

Si en $S u F$ se establecía que el conocimiento coincidía con la forma de la ciencia, en la $P S F$ se considerará que la forma y condiciones del acto de conocer, aunque se mantienen, ya no son características exclusivas de la ciencia. Ahora, esta forma peculiar de conocimiento se presenta como el primado epistemológico de la cultura humana. Cassirer, entonces, se esforzará en mostrar que una filosofía que investiga las formas simbólicas reconoce las distintas manifestaciones singulares como una expresión peculiar del mundo humano. Así, la cultura se convierte en el principio universal a través del cual se pueden valorar y asignar funcionalmente los índices particulares que permitirán identificar la singularidad como parte de una forma general o forma simbólica.

Una de las fuentes básicas a las que recurre Cassirer ( $P S F, \mathrm{I}, \mathrm{pp} .12-20)$ para fundar su teoría del símbolo es la teoría pictórica propuesta por Hertz (1899), específicamente en lo relacionado con la tesis que asume a las «imágenes o símbolos» como representaciones internas de los objetos externos del mundo:

We form for ourselves images or symbols of external objects and the form which we give them is such that the necessary consequents of the images in thought are always the images of the necessary consequents in nature of the things pictured. In order that this requirement may be satisfied, there must be a certain conformity between nature and our thought. Experience teaches us that the requirement can be satisfied, and hence that such a conformity does in fact exist (Hertz 1899, p. 1).

La relación entre las consecuencias intelectuales y naturales, plantea un realismo simbólico en el que la configuración del conocimiento opera como una «imagen o símbolo» de dicha relación consecuente entre lo intelectualmente estipulado y el conjunto de consecuencias naturales registradas ${ }^{16}$. Hertz (1899) considerará que

16 El trabajo de Andoni IBARRa y Thomas Mormann (2005) sobre las representaciones amplía el propio concepto de «representación» y, aunque evalúan las características de la ciencia como modelo paradigmático, toda su explicación permite una reflexión en torno a la teoría pictórica de Hertz y el eventual giro filosófico que Cassirer le imprime a esta teoría. A partir de este trabajo se puede sostener que el símbolo, no sólo es una isomorfía en donde existe semejanza especular entre el objeto y su imagen, sino que, a decir de los autores, el símbolo propuesto por el neokantiano, la relación de símbolo es funcional toda vez que el carácter relacional básico se constituye desde una vinculación lógica entre consecuencias intelectuales y naturales. Los autores, a partir de ello, distinguen los dominios simbólicos y empíricos para designar que el símbolo nunca es una suplantación de lo natural, sino su función lógica y, por tanto, la relación entre el constructo intelectual y el objeto percibido. Más adelante se retomará esta propuesta. 
bajo este principio de la vinculación funcional entre los postulados intelectuales y las manifestaciones naturales, operaba un principio explicativo que permitiría tanto la iteración de las imágenes entre sí para una mejor determinación de posibles futuros eventos, como la iteración del conocimiento que ofrecería una estructura cada vez más objetiva de los productos intelectuales en los que el investigador se apoyaba para la evaluación de sus modelos teóricos.

Cassirer asumirá este modelo pictórico propuesto por Hertz para sostener que la relación entre un evento específico y su organización epistemológica se debía al resultado de un proceso dialéctico. Para el neokantiano, era claro que dado un momento-A caracterizado por la percepción de un fenómeno cualquiera, se correspondía con un momento-B, el cual operaba como conceptuación del primero, de tal modo que en la relación de ambos puntos aparecía una función que expresaba su identidad. Para el de Breslavia, la percepción de los sucesos no se constituye como un momento cognoscitivo sino como su motivo básico y comprensible a la luz de una legalidad universal, por lo que entre A y B debe configurarse una «imagen o símbolo» intermedio que opere como el vínculo necesario entre el evento singular y el intelecto (SuF, pp. 309-349; PSF, I, pp. 290-310; PSF, II, pp. 51-88; PSF, III, pp. 331-416).

Con respecto a este punto anterior se ofrece el siguiente ejemplo que ilustra cómo la singularidad no se constituye como un momento que aparece, sino como el resultado de una relación de un modelo conceptual que permite la valoración de un hecho específico: "Los espasmos de la pierna de rana en el laboratorio de Galvani se convierten en prueba y testimonio de la nueva energía del "galvanismo» no como fenómenos en sí, inanalizables, sino en virtud del proceso analítico del pensamiento que llevan aparejado» ( $P S F, \mathrm{II}, \mathrm{p} .70)$. Lo singular se constituye no en virtud de su simple aparecer, sino en relación a una ley general que permite identificarlo como una particularidad específica de un sistema general.

En este punto, para profundizar este aspecto de la teoría funcional de Cassirer, conviene considerar el trabajo de Andoni Ibarra y Thomas Mormann (2005) quienes caracterizan la relación o representación simbólica de Hertz como una representación homológica, la cual, siguiendo a los autores, no dependen «ni de la semejanza objetual ni de la estructural. De ahí que se [aleje] de toda concepción de representación concebida como reflejo especular o basada en la noción de semejanza» (Ibarra y Mormann 2005, p. 13). Al analizar las propiedades de la representación propuesta por Hertz, los autores establecen al menos dos puntos importantes para nuestro trabajo: 1) que la relación entre un objeto-A y un objeto-B y su consecuente iteración, permite distinguir entre el dominio «simbólico» (representación) y el «empírico» (representación) de un modo natural; 2) que las relaciones entre objetos y su simbolización (relación iterada) puede interpretarse como una «representación lógica» lo que, para los autores, implica que «no son ni los objetos ni las estructuras los que son considerados y representados, sino únicamente las relaciones lógicas» (Ibarra y Mormann 2005, p. 18).

Andoni Ibarra (2015), en esta línea, ha profundizado estos resultados al explicar que la actividad representativa no se configura en una fidelidad a la realidad en un sentido especular, sino que, dado su carácter simbólico —es decir, en su dimensión relacional entre consecuencias lógicas entre naturaleza e intelecto- las representaciones ofrecen una mejor comprensión de los objetos y de su relación con el sujeto 
en la medida en que se acepta que el conjunto de prácticas culturales (teoría del arte, semiótica, epistemología, ciencias en general) ofrecen recursos novedosos de análisis para la comprensión de los procesos de conocimiento y representación en general.

Este resultado se puede encontrar también entre los teóricos interesados en estudiar específicamente la propuesta de Cassirer. Así, Sebastian Luft (2004) ha hecho notar que la filosofía de este autor se desarrolla en el marco de dos tradiciones básicas: la fenomenológica y la hermenéutica, lo que, a decir de Luft, permite que el neokantiano elabore una síntesis filosófica que tiene por objeto elucidar la interrelación entre el intelecto (mind) y el mundo (world) «This correlation gives an account of the way the world appears for an experiencing subject» (Luft 2004, p. 210. Énfasis en el original.).

Para Cassirer, la interrelación entre el sujeto y el objeto se logra por medio de una representación o simbolización del mundo ${ }^{17}$. El símbolo, en esta vinculación, no opera como una actividad que sustituye, sino como una actividad que re-presenta el modo en que el sujeto conoce, se vincula y experimenta el objeto. La singularidad no se sostiene como el resultado asilado de una advertencia fenoménica, sino como el consecuente de un contexto de significado en el que opera un principio o norma general que lo hace aparecer. Si tanto Luft como Ibarra han sido claros en exponer que la naturaleza de la representación se genera como el resultado de un proceso constructivo, esto quiere decir que el conocimiento singular es, en última instancia, el resultado de una representación del suceso particular a partir de un contexto general de significado. Lo singular no es lo que primariamente experimentamos, sino el universal el que ofrece las posibilidades de experiencia del singular.

Con lo dicho, se puede señalar un primer resultado: el universal es la condición del singular toda vez que a partir de una forma simbólica se ofrecen las condiciones de identificación entre fenómenos y de individualidades separables en relación a un sistema general de sentido. Con este aspecto logrado, se vuelve necesario profundizar en la naturaleza de la representación para sostener que, a partir de un sistema simbólico es posible construir una relación de identidad entre el símbolo y el evento empírico. Específicamente se debe explicar ¿Bajo qué mecanismos la función permite la vinculación entre los aspectos universal y singular? ¿Cuáles son los recursos que permiten relacionar esta universalidad con el conjunto de singularidades que advertimos cotidianamente?

${ }_{17}$ Sobre los conceptos sujeto, objeto y percepción, se puede consultar los distintos estudios que integran su Fenomenología del conocimiento, especialmente: «Análisis subjetivo y objetivo» (PSF, III, pp. 61-75), «Cosa y atributo» (PSF, III, pp. 143-170); «La preñez simbólica» (PSF, III, pp. 226-240), «Concepto y objeto» (PSF, III, pp. 368-383). Especialmente en estos pasajes, el autor, sin llegar a fijar una definición puntual, establece que el ser humano, en tanto que capaz de simbolización, es el sujeto que conoce la realidad captada a través del conjunto de sus signos físicos. La tesis del autor es que la iteración permite la construcción de conceptos simbólicos. De este modo, el sujeto no construye al objeto solo lo advierte como «cosa» de la cual es posible «atribuirle» determinadas características. Cassirer ofreció un resumen de su filosofía en la que él puntualiza que el ser humano, como animal simbólico, es el agente constructor de la cultura por medio de la cual accede al conocimiento de la realidad y, por tanto, de la verdad: $(A E M)$. 
Parte de la explicación se encuentra en la filosofía de Cassirer (PSF, I, pp. 15ss.) quien considera que la función matemática, como principio de relación, permite la construcción de conceptos que describan la relación entre los objetos concretos y una cierta modalidad de interdependencia. Esta forma de conocimiento se logra sólo en la medida en que estas formaciones conceptuales pertenezcan desde un principio a una determinada perspectiva unitaria del conocimiento. Para el neokantiano es solo en el marco de ciertas perspectivas peculiares que constituyen a cada forma estudiada como se puede construir el conocimiento. No resulta extraño que cada volumen de la Filosofía de las formas simbólicas se refiera a un problema peculiar del conocimiento y represente una forma específica pero que, en su conjunto, contribuyen a la investigación general del Ser (PSF, III, pp. 12-17).

Este aspecto ha sido resaltado por Thomas Ryckman (1991) quien considera que el principal fundamento metodológico de la función en el neokantiano se encuentra en el establecimiento de una forma o ley general que permite evaluar el conjunto de unidades singulares que se hacen presentes al sujeto. El autor considera que la teoría de Cassirer asume el singular como «signo» que no se reduce a las unidades físicas aun cuando se perciban como resultado de una interacción operativa cotidiana; de este modo, la luz, el agua, el sonido o, incluso, actividades más complejas como la organización política de una sociedad, no pueden asumirse como acciones aisladas, sino que pueden considerarse «luz», «agua», «sonido» o «actividades políticas» en la medida en que estas acciones unitarias se valoren desde una expresión universal que permita designarlas como tales. Ryckman escribe:

Objectivity requires that the intellectual transformation of a group of phenomena in the formation of the scientific concept be effected according to "certain permanent relations in the flux of sensations», the «rules» of which "we can call to mind independently of the change of the momentary material». Indeed, only on this basis is a «scientific concept of Nature developed and confirmed» ( Ryckman, 1991, p. 72).

Este texto se apoya de un texto esencial de $S u F$ en el cual se hace explícita la teoría constructivista del neokantiano ${ }^{18}$. El sentido general en ambos textos postula que la actividad teórica no busca la explicación de los elementos singulares que se presentan a los sentidos aduciendo una relación entre el suceso singular con una forma general que les dote de significado. La propuesta de lectura que ofrece

18 Ryckman está considerando el siguiente texto de Cassirer: «The conception of the unambiguity and "stability" of being obviously is not in the content of perception as given in our first immediate experience, but it indicates the goal, which the intellectual labor of science seeks to have this content approximate. This goal can only be reached, if we are able to establish certain permanent relations in the flux of sensations, which differ and have their truth limited to a single moment of time; the rules of these permanent relations we can call to mind independently of change of the momentary material» (SuF, p. 264). El contexto general del texto corresponde a una valoración que el neokantiano realiza en torno a si la construcción de la función se debe a una supeditación de las «representaciones» (Vorstellungen), la «realidad» (Wirklichkeit) o si, por el contrario, se debe asumir que ya el propio concepto de «realidad» presupone un sistema de postulados intelectuales previos. Para Cassirer la "estabilidad» del conocimiento no se puede asumir como un fundamento sino como una meta a la cual llegar. 
Ryckman (1991, pp. 62-75), hace notar que para Cassirer la objetividad y, por tanto, el conocimiento tiene como punto de origen la consideración de una ley que permite evaluar las expresiones culturales como fenómenos singulares. Bajo esta perspectiva, el singular no es el punto de partida sobre el cual se debe reflexionar, sino la relación funcional entre una forma general de sentido y su incorporación una forma de expresión peculiar.

Esto anterior se aprecia mejor en el planteamiento del problema propuesto en $P S F$, III (pp. 35-50), en donde Cassirer estipula que, la consideración de los productos de la sensación como puntos de partida sólidos para la construcción de una teoría del conocimiento, acarrean el problema de cómo ha sido posible siquiera la construcción de un marco teórico que permita sostener que los productos de la sensibilidad son objetos válidos de conocimiento. Para el autor, el problema de lo concreto, de las cosas físicas, se resuelve por el estudio de los medios que hacen posible que los objetos se asuman como tales, así como de las leyes que nos vinculan a las singularidades. Bajo este marco expuesto, el neokantiano entenderá como problema de partida, que la singularidad no es una característica de la realidad, sino un resultado peculiar del proceso del conocimiento. Para el neokantiano, el conocimiento del singular es el consecuente de un proceso de relación que inicia con una «coherencia unitaria que va desde el mero valor expresivo de la percepción y desde el carácter representativo de la representación [...] hasta las significaciones universales del lenguaje y del conocimiento teorético» $\left(P S F\right.$, III, p. 57) ${ }^{19}$.

Según Cassirer lo singular no es un problema que se hace presente como unidad aislada de una forma que permite asumirlo como "apariencia», "problema», o «conocimiento», sino que se refiere al producto de una identidad entre el pensamiento con la realidad por medio de formaciones simbólicas que permiten dotar de significado a cada miembro de la serie como perteneciente a una forma peculiar de significación:

19 Esta afirmación de Cassirer puede acentuarse mejor a la luz de ciertos problemas computacionales actuales. Mientras que para el neokantiano el interés está en cómo la percepción permite una aproximación funcional a la realidad, para los investigadores en materia computacional, el objeto de interés es construir entornos virtuales que aumenten la experiencia de la percepción. El acento, entonces parte de polos contrarios, en el caso de la filosofía, la realidad se asume como un factor por adecuar y se investiga el tipo de relación que puede ofrecer la percepción para llegar a ella, en la computación la realidad, al ser evidente, asume como problema a la percepción como la meta por lograr. Así, los problemas son opuestos, la filosofía considera que se debe estudiar el medio para adecuar la percepción con la realidad, en computación, la pregunta es cómo la realidad permite ajustar su percepción. Según FLORIDI (2017, pp. 287-293), en este sentido, también reconoce que, a partir de las Ciencias computacionales, específicamente desde los Métodos Formales, se pueden desarrollar distintos estudios experimentales (por ejemplo, evaluar el funcionamiento neuronal a partir de ciertas simulaciones algorítmicas simuladas en una computadora) que se asumen como conocimiento válido. La razón central de esta validación es porque «la relación de simulación y la relación de realización son equivalentes porque son relaciones que describen los mismos procesos» (FLoRIDI 2017, p. 292). Es decir, la investigación filosófica se ha visto nutrida a partir de aportaciones computacionales, asumiendo como base que la percepción de un funcionamiento simulado ofrece validez a la percepción de un funcionamiento real (experimental). Para un estudio de estos problemas: $c f r$. Delgado-Mata e Ibañez, (2006). 
Toda determinación y dominio teóricos del ser dependen de que el pensamiento, en lugar de vérselas directamente con la realidad, elabore un sistema de signos y aprenda a utilizar estos signos como representantes de los objetos. A medida que esta función de representación se vaya imponiendo, el ser empieza a transformarse en un todo ordenado, en un contexto que se puede abarcar claramente con la mirada. El ser y el acaecer particulares se ven infiltrados cada vez más por determinaciones generales a medida que se logre representar su contenido de esta manera. Siguiendo estas determinaciones y representado a su vez simbólicamente cada una de ellas, el pensamiento logra un modelo cada vez más perfecto del ser y de su estructura teórica total (PSF, III, p. 61).

Para el filósofo de Breslavia, esta función que asume la representación de los objetos como signos de cosa o de expresiones, no es una representación por «sustitución» ${ }^{20}$, sino una relación simbólica en la que el objeto representado se conoce por su característica relacional. En este carácter representativo, no se suplanta el signo por el objeto prevaleciendo la «idea» en lugar de la «realidad», sino

20 Así caracteriza Fernando Zamora la representación propuesta por Cassirer, como representación sustitutiva: «Aquí pretendo mostrar que este filósofo [neokantiano] concibe la representación en términos sígnico-simbólicos, es decir, en un sentido que podría ser fundamentalmente espacial. Para ello tomaré como criterio esta definición de representación sustitutiva: algo es la imagen de otra cosa, o la representa, cuando puede estar en su lugar, debido a que puede sustituirla» (ZAMORA 2013, pp. 270-271. Énfasis en el original.). Este aspecto es acentuado cuando el autor plantea que Cassirer «entiende el acto representativo como una creación de sustitutos ¿Por qué este juguete cumple tan bien con su papel de representar a un caballo verdadero? ¿Cómo funcionan en general los juguetes?» (Ibid., p. 274). No me puedo extender en la explicación, pero en definitiva esta es una interpretación incorrecta de la representación en el neokantiano, para quien, la representación no se reduce a considerar a un juguete como si fuera un caballo, sino que, según la propuesta del neokantiano, se debe asumir al «juguete» como un caballo verdadero. Según el filósofo de Breslavia, la relación que permitiría asumir un «objeto-juguete» como un "caballo verdadero», no son las reglas de un juego ni las posibilidades representativas alegóricas, sino las condiciones sociales de la comunidad; las posibilidades representativas están cargadas, entre otros aspectos, por la forma de la vida (PSF, I, pp. 195-286), así como de la actividad cultural (AEM, pp. 278-286). Como argumento, reproduzco el siguiente pasaje para mostrar que la representación en Cassirer no es una sustitución, sino una presencia: "La "imagen" no representa la "cosa"; es la cosa; no sólo la representa, sino que opera como ella sustituyéndola en su inmediato presente. Consiguientemente, bien puede ser un índice de que el pensamiento mitológico carece de la categoría de lo "ideal" y, por tanto, siempre que se topa con algo puramente significativo, para aprehender esta significación pura tiene que transformarla en un ser o cosa. Esta misma relación básica aparece en las más diversas etapas del pensamiento mítico, pero mucho más claramente que en el mero pensamiento está expresada en la actividad mítica... ninguno de los ritos tiene originalmente un sentido meramente alegórico, imitativo o representativo, sino siempre real; están entrelazados con la realidad de la acción de tal modo que forman una parte integrante indispensable de ella» ( $P S F$, II, p. 63. Énfasis en el original). Se podría pensar que esta característica presentativa (y no sustitutiva como propone Zamora) correspondería a una característica particular de la función expresiva propia del mito, pero es posible encontrar este mismo rasgo representativo en todas las formas consideradas por este autor ( $P S F$, I, pp. 12-60; PSF, III, pp. 59-127; PSF, IV, pp. 3-33). Para un tratamiento sistemático de la función en este autor y una valoración más amplia de este punto: EsPARzA 2017, pp. 96-154). 
que se mantiene la identidad entre el sujeto y el objeto. El ser humano, en este contexto, percibe la vida como experiencia vital global y sólo en un paulatino proceso de autoconocimiento, el individuo avanza de la advertencia de la totalidad de la percepción como experiencia homogénea, hacia la diferenciación progresiva de los fenómenos individuales, sin con ello perder el punto de vista unitario a través del cual, las formas particulares que revelan y expresan esa misma unidad, se constituyen como formas particulares de la forma general de la serie.

Es bajo este marco conceptual en el que el neokantiano soporta su filosofía de las formas simbólicas; al considerar que las unidades singulares aparecen en un contexto de significado específico (Hartman, 1949) y que, a partir de este medio de relación se desarrolla una operación funcional. En este punto, aparecen nuevos sistemas representativos que, aunque no operen con las demandas propias de la Ciencia, satisfacen el conjunto de criterios propios de un sistema de relaciones (Ibarra y Mormann, 2005). Para Cassirer, la filosofía de las formas simbólicas amplía tanto el contenido como el método para comprobar que «no sólo son auténticos factores y motivos formales los que imperan en la configuración de la imagen científica del mundo, sino también los que ya existen en la configuración de la "imagen natural del mundo", la imagen de la percepción y la intuición» ( $P S F$, III, p. 7).

Todo sistema simbólico opera bajo el interés de considerar la realidad y el continuo de objetos singulares como expresiones formales o signos de distintas formas culturales, por lo que se debe valorar cada expresión bajo un «índice de refracción particular». La tarea que a la filosofía le corresponde, según el neokantiano, es conocer «la naturaleza específica de los distintos medios de refracción; [la filosofía] quiere comprender cada uno de ellos en cuanto a su composición y en cuanto a las leyes de su estructura» (PSF, III, p. 12). En este sentido, cada forma específica bajo una directriz propia; así, el mundo mitológico, opera expresivamente ( $P S F, \mathrm{II})$, mientras que el lenguaje lo realiza de forma representativa $(P S F, \mathrm{I})$, para finalmente, lograr una representación significativa $(P S F, \mathrm{III})^{21}$.

$\mathrm{Si}$ valoramos estas reflexiones en el marco de nuestro problema, se puede apreciar que el proceso básico propuesto por Cassirer plantea que por medio de la relación funcional de los datos singulares con alguna forma general posibilita, primero, el reconocimiento de la singularidad y, segundo, su tratamiento a la luz de la forma general considerada. El neokantiano acepta que a partir de las formaciones simbólicas, el conocimiento del singular ya no se ofrece como semejanza de contenido entre el concepto universal con el que se significa lo particular, sino como relación formal entre el concepto general y el singular conocido. En este sentido, las formas simbólicas consideradas por Cassirer, vinculan desde un punto de vista peculiar con la unidad en virtud de una simetría entre la forma intelectual y el particular del mundo natural que pretende significar, relaciona al singular con el Universal por medio de la ley peculiar que a cada forma corresponde.

${ }^{21}$ Por ahora no se puede ofrecer una interpretación más extensa de las distintas formas simbólicas. Para una valoración crítica de aquellas se recomienda el trabajo de González (2013, pp. 73-120) y ESPARZA (2017, pp. 189-290). 


\section{CONCLUSIONES}

En el presente artículo se elucidaron las relaciones entre el singular y el universal (S/U) en la filosofía de Ernst Cassirer. Como problema de partida se fijó que la individualidad no puede ser conocida sin antes ofrecer una virtud intelectual que relacione la singularidad con una forma de conocimiento general que permita designar la unidad como una actividad epistemológica vinculada. Para sostener esta hipótesis, se estableció que la Philosophie der Symbolischen Formen de Ernst Cassirer permitía lograr el objetivo propuesto y se sostiene que: 1) la percepción de una experiencia individual no es el punto de partida para la construcción del conocimiento sino su motivo; 2) todo conocimiento es una relación entre una forma general o ley que permite identificar, dentro del conglomerado de fenómenos de la realidad, a los miembros singulares de una forma; 3) a partir de esta identificación, es posible establecer la singularidad ya no como un problema sino como un producto específico de una relación entre una forma de la serie y los miembros de que corresponden a esa serie; 4) Cassirer, luego de su obra magna ( $P S F$, ampliará el concepto de teoría aceptando otras formaciones espirituales que cumplían con las características representativas en que se fundaba la ciencia, de este modo, la crítica del conocimiento reconoce a las formas epistemológicas puras o simbólicas, y acepta que tanto el conocimiento expresivo (mito) como el representativo (lenguaje) ofrecen medios para conocer la singularidad a partir de distintas formaciones simbólicas.

El conjunto de resultados que aquí se presentan tiene como interés futuro resolver un problema mayor que refiere a los mecanismos por medio de los cuales es posible el autoconocimiento. La principal tensión conceptual establece que, si el conocimiento mantiene como característica primera la «universalización» de las unidades particulares, entonces, el conocimiento del "yo», debe resolver cómo la cognición del ser personal armoniza con el conjunto de características generales en las que se soporta la individualidad. Los resultados aquí alcanzados, permitirán sostener que: el autoconocimiento del yo (singular) es universal gracias a las formas simbólicas en las que se apoya para fundar el conjunto de predicaciones a través de las cuales se caracteriza al individuo. El «yo» es cognoscible en virtud de las formas culturales en las que se apoya para autoconceptuarse como ser individual.

\section{BiBLIOGRAFÍA}

Cassirer, E. (1902). Leibniz' System in seinen wissenschaftlichen Grundlagen. Marburgo, N. G. Elwert'sche Verlagsbuchhandlung

- (1910). Substanzbegriff und Funktionsbegriff. Untersuchungen über die Grundfragen der Erkenntniskritik. [(1953). Substance and Function. Traducción al inglés de W.C. Swabey \& M.C. Swabey. New York: Dover Publication].

- (1923). Philosophie der symbolischen Formen: Die Sprache. Berlín: Bruno. [(1998). Filosofía de las formas simbólicas: El lenguaje. Traducción al español de Fernando Morones. México: Fondo de Cultura Económica]. 
- (1925). Philosophie der symbolischen Formen: Das Mythische Denken. Berlín: Bruno. [(1998). Filosofía de las formas simbólicas: El pensamiento mítico. Traducción al español de Fernando Morones. México: Fondo de Cultura Económica].

- (1929). Philosophie der symbolischen Formen: Phänomenologie der Erkenntnis. Berlín: Buruno Cassirer. [(1998). Filosofía de las formas simbólicas. Fenomenología del conocimiento. Traducción al español de Fernando Morones. México: Fondo de Cultura Económica].

- (1945). An Essay on Man: an Introduction to a Philosophy of Human Culture. New York: Doubleday Anchor.

- (1975). La filosofía de la ilustración. Traducción al español de: Eugenio Ímaz. México: Fondo de Cultura Económica.

- (1979). Symbol, Myth and Culture. Essays and lectures of Ernst Cassirer (1935-1945). Editado por D. Verene. New Haven and London: Yale University Press.

- (1993). El problema del conocimiento, Vol. 1. Traducción al español de Wenceslao Roces. México: Fondo de Cultura Económica.

- (1995). Zur Metaphysik der symbolischen Formen. Edición a cargo de John Michael Krois. Felix Meiner. [(1996), The Philosophy of Symbolic Forms: The Metaphysics of Symbolic Forms. Traducción al inglés de J.M. Krois. Edición a cargo de J.M. Krois y D. P. Verene. New Haven: Yale Univesity].

Delgado-Mata, C.; Ibañez, J., Eds. (2006). Proceedings of the IVEVA 2006: international conference, México: Ed. Los libros de Homero.

Esparza, G. (2017). La construcción simbólica de sí mismo. Madrid: Editorial Académica Española.

- (2019). «Forma y legalidad del conocimiento mítico en Ernst Cassirer». En: Calabrese, C.; Esparza, G.; Junco, E. Mito, conocimiento y acción. Continuidad y cambio en los procesos culturales. Londres/Nueva York: Peter Lang, 41-73.

Ferrari, (2010). «Is Cassirer a neokantian methodologically speaking?». En: Makkreel, Rudolf \& LUFT, Sebastian, Neokantianism in Contemporary Philosophy, Bloomington \& Indianapolis: Indiana University Press, 2010, 293-314.

Floridi, L. (2017). «Una defensa del construccionismo: la filosofía como ingeniería conceptual». Pensamiento: Revista de Investigación e información filosófica., Vol. 73, No. 276: 271-300.

García, M. (1998). «La cultura como universo simbólico en la antropología de E. Cassirer». Pensamiento: Revista de Investigación e información filosófica. Vol. 54, No. 209: 221-244.

Gawronsky, D. (1949). «Ernst Cassirer: his life and his work». En: Schilpp, P (1949): 1-37.

González, R. (2013). Renovación del humanismo y emancipación antropológica. Hacia una metafísica del umbral a partir de la filosofía de las formas simbólicas. México: Fontamara.

- (2015). Eduardo Nicol y Ernst Cassirer: antropología y ontología. Del animal simbólico a la idea del hombre como ser de la expresión. Madrid: Editorial Académica Española.

Hartman, R. (1949). "Cassirer's Philosophy of Symbolic Forms». En Schilpp, P. (1949): 289-333.

Hertz, H. (1899). The principles of Mechanics presented in a new form. Traducido al inglés por D. Jones \& J. Walley. Londres: Macmillan and Co.

Ibarra, Andoni \& Mormann, Thomas. (2000). Una teoría combinatoria de las representaciones científicas. Revista hispanoamericana de filosofía, vol. 32, no. 95: 3-46.

- (2015). «La representación». Cátedra Ezequuiel A. Chávez. Aguascalientes: Universidad Autónoma de Augascalientes.

Ikonen, S. (2011). "Cassirer's critique of culture. Between the Scylla of Lebensphilosophie and the Charybdis of the Vienna Circle». Synthese, Vol. 179: 187-202.

Kauffman, F. (1949). "Cassirer's Theory of Scientific Knowledge». En: Schilpp (1949): 183-214. 
Krois, J. (2011). «The priority of symbolism over language in Cassirer's philosophy». Synthese, Vol. 179: 9-20.

Luft, S. (2004). «A Hermeneutic Phenomenology of subjective and objective Spirit: Husserl, Natorp and Cassirer», The new yearbook of phenomenology and phenomenological Philosophy, Vol. 4, 209-248.

Orth, E. (2011). «Ernst Cassirer as cultural Scientist». Synthese. Vol. 179: 115-134.

Rosenstein, L. (1973). «Some metaphysical problems of Cassirer's symbolic Forms», Man and World, Vol. 6, No. 3, pp. 304-321.

Ryckman, T. (1991). Conditio sine qua non? Zuordnung in the early epistemologies of Cassirer and Schlick. Synthese, Vol. 81: 57-95.

Schilpp, P. (1949). The Philosophy of Ernst Cassirer, Vol. VI. Evanston, Illinois: The Library of living philosophers, Inc.

Verene, D. (2008). «Cassirer's Metaphysics». En: Barash, J., The symbolic construction of reality: The legacy of Ernst Cassirer, Chicago: Chicago University press, 93-123.

Zamora, F. (2007). Filosofía de la imagen. Lenguaje, imagen y representación. México: Universidad Autónoma de México.

Universidad Panamericana

Gustavo Esparza

Aguascalientes, México

gaesparza@up.edu.mx

/ https://orcid.org/0000-0002-9470-6519

[Artículo aprobado para publicación en enero de 2018] 